\title{
Effect of Brazilian Propolis on Exacerbation of Respiratory Syncytial Virus Infection in Mice Exposed to Tetrabromobisphenol A, a Brominated Flame Retardant
}

\author{
Tomomi Takeshita, ${ }^{1}$ Wataru Watanabe, ${ }^{2}$ Satomi Toyama, ${ }^{1}$ Yuya Hayashi, ${ }^{1}$ Shiori Honda, ${ }^{1}$ \\ Shuichi Sakamoto, ${ }^{1}$ Sayuri Matsuoka, ${ }^{1}$ Hiroki Yoshida, ${ }^{1}$ Shiro Takeda, ${ }^{3}$ Muneaki Hidaka, \\ Shigetoshi Tsutsumi, ${ }^{5}$ Ken Yasukawa, ${ }^{6}$ Yong Kun Park, ${ }^{7}$ and Masahiko Kurokawa ${ }^{1}$ \\ ${ }^{1}$ Department of Biochemistry, Graduate School of Clinical Pharmacy, Kyushu University of Health and Welfare, 1714-1 Yoshino, \\ Miyazaki, Nobeoka 882-8508, Japan \\ ${ }^{2}$ Department of Microbiology, Graduate School of Clinical Pharmacy, Kyushu University of Health and Welfare, 1714-1 Yoshino, \\ Miyazaki, Nobeoka 882-8508, Japan \\ ${ }^{3}$ Research Division, Minami Nihon Rakuno Kyodo Co. Ltd., Miyazaki, Miyakonojo 885-0003, Japan \\ ${ }^{4}$ Clinical Pharmacy, Graduate School of Clinical Pharmacy, Kyushu University of Health and Welfare, 1714-1 Yoshino, \\ Miyazaki, Nobeoka 882-8508, Japan \\ ${ }^{5}$ Amazonfood Ltd., 3-1-8 Misaki, Chiyoda, Tokyo 101-0061, Japan \\ ${ }^{6}$ School of Pharmacy, Nihon University, 7-7-1 Narashinodai, Chiba, Funabashi-shi 274-8555, Japan \\ ${ }^{7}$ Department of Food Science, College of Food Engineering, State University of Campinas, P.O. Box 6177, \\ 13083-970 Campinas, SP, Brazil
}

Correspondence should be addressed to Masahiko Kurokawa; b2mk@phoenix.ac.jp

Received 3 June 2013; Accepted 14 September 2013

Academic Editor: F. R. F. Nascimento

Copyright (C) 2013 Tomomi Takeshita et al. This is an open access article distributed under the Creative Commons Attribution License, which permits unrestricted use, distribution, and reproduction in any medium, provided the original work is properly cited.

\begin{abstract}
Tetrabromobisphenol A (TBBPA), a brominated flame retardant, has been found to exacerbate pneumonia in respiratory syncytial virus- (RSV-) infected mice. We examined the effect of Brazilian propolis (AF-08) on the exacerbation of RSV infection by TBBPA exposure in mice. Mice were fed a powdered diet mixed with $1 \%$ TBBPA alone, $0.02 \%$ AF-08 alone, or $1 \%$ TBBPA and $0.02 \%$ AF-08 for four weeks and then intranasally infected with RSV. TBBPA exposure increased the pulmonary virus titer and level of IFN- $\gamma$, a representative marker of pneumonia due to RSV infection, in the lungs of infected mice without toxicity. AF- 08 was significantly effective in reducing the virus titers and IFN- $\gamma$ level increased by TBBPA exposure. Also, AF-08 significantly reduced proinflammatory cytokine (TNF- $\alpha$ and IL-6) levels in the lungs of RSV-infected mice with TBBPA exposure, but Th2 cytokine (IL-4 and IL-10) levels were not evidently increased. Neither TBBPA exposure nor AF-08 treatment affected the anti-RSV antibody production in RSV-infected mice. In flow cytometry analysis, AF-08 seemed to be effective in reducing the ratio of pulmonary $\mathrm{CD}^{+} \mathrm{a}^{+}$cells in RSV-infected mice with TBBPA exposure. TBBPA and AF-08 did not exhibit anti-RSV activity in vitro. Thus, AF08 probably ameliorated pneumonia exacerbated by TBBPA exposure in RSV-infected mice by limiting excess cellular immune responses.
\end{abstract}

\section{Introduction}

Propolis has been used worldwide as a folk medicine since ca. $300 \mathrm{BC}$ and as a dietary supplement to maintain or improve human health [1-3]; it is currently used as an alternative medicine in the management of various ailments [4]. Propolis is a resinous hive product and consists of a mixture of plant exudates collected by honeybees and beeswax. The ethanol extract of Brazilian propolis (AF-08) has been shown to exhibit anti-influenza virus activity in vitro and in vivo and 
ameliorate influenza symptoms in mice [5]. We also showed that AF-08 has an immunomodulatory activity against intradermal herpes simplex virus type-1 (HSV-1) infection in mice and ameliorates herpes symptoms in mice, although it has no anti-HSV-1 activity in vitro [6]. The immunomodulatory activity associated with interferon- (IFN-) $\gamma$ production inducing Thl immunity in mice was suggested to contribute to the elucidation of various pharmacological actions of propolis in health and disease [6].

Human respiratory syncytial virus (RSV), a member of the Paramyxoviridae family, is the most prevalent infectious agent of acute lower respiratory illness in infants and young children [7]. Infection and reinfection with RSV are frequent during the first few years of life, and most children are infected by 24 months of age [8]. Clinically severe RSV infection is seen primarily in young children with naive immune systems and/or genetic predisposition [9], patients with suppressed T-cell immunity [7], and the elderly [10]. It is suggested that the severity of RSV infection is associated with immunological defect.

We have recently established a novel assay system for the evaluation of the developmental immunotoxicity of brominated flame retardants (BFRs) using a mouse model of RSV infection [11]. Using this model, perinatal exposure to BFRs has been shown to elevate the levels of IFN- $\gamma$ in the bronchoalveolar lavage fluid (BALF) of RSV-infected offspring mice with an increase in pulmonary viral titers and exacerbate pneumonia $[12,13]$, indicating that BFRs are a risk factor for RSV infection across human generations. BFRs are ubiquitously used as industrial materials worldwide. They are used as additive or reactive components in a variety of polymers, such as polystyrene foams, high-impact polystyrene, and epoxy resins [14]. Notably, tetrabromobisphenol A (TBBPA) accounted for approximately $76 \%$ of the BFRs consumed in Asia in 2001, and the amount used was approximately 90,000 tons [14]. BFRs are easily released into an environment due to deterioration or abrasion of the materials and exist ubiquitously in the environment [1517]. Some of them have been shown to cause endocrine disruption and reproductive damage and to be immunotoxic and neurotoxic [18-23]. They are suspected of being toxic to children [24]. Recently, we demonstrated changes in cytokine production and immune cell populations not only in offspring mice born to mice perinatally exposed to BFRs but also in normal mice exposed to BFRs, and the irregularities due to BFRs exposure have been suggested to exacerbate pneumonia in RSV-infected mice [25].

In this study, the effect of Brazilian propolis (AF-08) on the exacerbation of RSV infection in mice exposed to TBBPA was examined. We found that AF-08 alleviated the symptoms of pneumonia exacerbated by TBBPA exposure. The mode of alleviation was evaluated virologically and immunologically, and we characterized the potential and pharmacological activity of Brazilian propolis AF-08.

\section{Materials and Methods}

2.1. Cell and Virus. Human epidermoid carcinoma (HEp-2) cells (American Type Culture Collection, CCL-23, Rockville,
MD) were purchased from Dainippon Pharmaceutical (Osaka, Japan) and grown and maintained in Eagle's minimum essential medium supplemented with $10 \%$ and $2 \%$, respectively, heat-inactivated fetal calf serum. The A2 strain of RSV was obtained from American Type Culture Collection (Rockville, MD) and grown in HEp-2 cell cultures. Viral titers of HEp-2 cell cultures were measured by the plaque method and expressed as plaque-forming units per milliliter (PFU/mL) [11].

2.2. TBBPA and Propolis. TBBPA (Mw: 543.87; purity: >93\%) was purchased from Tokyo Kasei (Tokyo, Japan) and mixed into a powdered diet, which was soy-free to avoid the estrogen-like effect of soybeans, based on the formulation of the NIH-07 open-formula rodent diet [26] and produced by Oriental Yeast Co. (Chiba, Japan).

Brazilian propolis AF0307 was immersed in 95\% ethanol $(1: 1, \mathrm{w} / \mathrm{w})$ for 3 months with occasional mixing at room temperature in the dark to avoid UV/light exposure, and the ethanol extracts were dried in a vacuum oven at $37^{\circ} \mathrm{C}$ to $38^{\circ} \mathrm{C}$ for 40 to $60 \mathrm{~min}$ as described previously [6]. The voucher specimen of AF0307 was deposited at Amazonfood Co., Ltd., Tokyo, Japan. The ethanol extract (AF-08) was supplied by Amazonfood Co., Ltd., Tokyo, Japan. AF-08 was dissolved in dimethyl sulfoxide (DMSO) at $100 \mathrm{mg} / \mathrm{mL}$ as a stock solution and diluted with culture medium to make various final concentrations for in vitro assays. The concentration of DMSO in each medium was less than $0.2 \%$. For in vivo assays, a powdered soy-free diet that had already been mixed with $1 \%$ TBBPA (w/w) was further mixed with AF-08 at $0.02 \%(w / w)$.

2.3. Mice. Female BALB/c mice ( 4 weeks old) were purchased from Kyudo Animal Laboratory (Kumamoto, Japan). The mice were housed 5 to 7 per cage in specific pathogen-free conditions, with a conventional solid diet CRF-1 (Oriental Yeast Co.) and water ad libitum and under a $12 \mathrm{~h}$ light $/ 12 \mathrm{~h}$ dark diurnal cycle (light at 7.00 a.m.). The temperature in the room was kept at $24 \pm 2^{\circ} \mathrm{C}$. The mice were acclimated for 7 days before starting experimental procedures. Animal studies followed the animal experimentation guidelines of Kyusyu University of Health and Welfare and were carried out in an approved biosafety level facility.

2.4. Murine RSV Infection. Five-week-old mice were fed a soy-free diet mixed with $1 \%(\mathrm{w} / \mathrm{w})$ TBBPA alone, $0.02 \%$ (w/w) AF-08 alone, or 1\% TBBPA and 0.02\% AF-08 for 4 weeks. As a control, the mice were fed a soy-free diet without $1 \%$ TBBPA and $0.02 \%$ AF-08. After treatment, these mice were fed CRF-1 and used for the subsequent RSV infection experiments. Throughout the experiments, both chow and water were provided for ad libitum consumption. The RSV infection was performed as reported previously [25]. Briefly, 9-week-old female mice were infected intranasally with $1 \times 10^{6}$ PFU per $0.1 \mathrm{~mL}$ of RSV under anesthesia. Mockinfected mice were also inoculated intranasally with $0.1 \mathrm{~mL}$ of phosphate-buffered saline (PBS) under anesthesia. On day 5 after infection, sera were obtained from the mice under anesthesia, and BALF was also obtained from the mice by 
instilling $1.0 \mathrm{~mL}$ of cold PBS into the lungs and aspirating it from the trachea using a tracheal cannula on days 1 and 5 after infection [27]. Ice-cold BALF was centrifuged at $100 \times \mathrm{g}$ at $4^{\circ} \mathrm{C}$ for $10 \mathrm{~min}$. After centrifugation, the supernatant was stored at $-80^{\circ} \mathrm{C}$ until use. The cell pellets were suspended in PBS on ice and used as bronchoalveolar lavage (BAL) cells for flow cytometric analysis. For virus titration, the lungs were removed on day 5 , immediately frozen in liquid $\mathrm{N}_{2}$, and stored at $-80^{\circ} \mathrm{C}$. Frozen lung tissue was homogenized with cold quartz sand in a homogenizer, and viral titers in the supernatants of the homogenates were measured by a plaque assay [25].

2.5. Plaque Reduction Assay and Cytotoxicity Assay. The antiRSV activities of TBBPA and AF-08 were examined by a plaque reduction assay using HEp-2 cells [11]. Briefly, HEp-2 cells grown in 24-well plates were infected with $100 \mathrm{PFU} / 0.2 \mathrm{~mL}$ of $\mathrm{RSV}$ at $37^{\circ} \mathrm{C}$ for $1 \mathrm{~h}$. The cells were overlaid with $1 \mathrm{~mL}$ of maintenance medium containing $0.8 \%$ methylcellulose and various concentrations of TBBPA, AF08 , or ribavirin and maintained in a humidified atmosphere containing $5 \% \mathrm{CO}_{2}$ for $4-5$ days. The infected cells were fixed and stained, and the plaques were counted [11]. TBBPA and AF-08 were dissolved in DMSO and diluted with culture medium to make the various final concentrations. The concentration of DMSO in each medium was less than $0.5 \%$. Ribavirin was dissolved in distilled water and used as a control. In the plaque reduction assay, the visible cytotoxicity of TBBPA and AF- 08 was evaluated by the extent of absence of uninfected cells on the surface of stained dishes, and strong cytotoxicity $(++)$ was scored as the absence of more than $50 \%$ of uninfected cells as compared with untreated dishes (controls) as described previously [28].

Cytotoxicities of TBBPA, AF-08, and ribavirin were assessed by MTT (3-[4,5-dimethylthiazol-2-yl]-2,5-diphenyltetrazolium bromide, Sigma, Tokyo, Japan) assay using HEp-2 cells in 96-well plates as described previously [29]. Briefly, HEp-2 cells grown in 96-well plates were overlaid with $50 \mu \mathrm{L}$ of maintenance medium containing various concentrations of the compounds and maintained in a humidified atmosphere containing $5 \% \mathrm{CO}_{2}$ for 3 days. TBBPA and AF08 were dissolved in DMSO and diluted with culture medium to make the various final concentrations. The concentration of DMSO in each medium was less than $0.5 \%$. Ribavirin was dissolved in distilled water and used as a control. For the MTT assay, $10 \mu \mathrm{L}$ of MTT $(7.5 \mathrm{mg} / \mathrm{mL})$ solution in PBS was added to each well and incubated for $4 \mathrm{~h}$ at $37^{\circ} \mathrm{C}$. The crystallized formazan in the plates was dissolved by the addition of $100 \mu \mathrm{L}$ of $20 \%$ (w/v) SDS/50\% (v/v) $\mathrm{N}, \mathrm{N}$-dimethylformamide. Absorbance was measured at two wavelengths (540 and $690 \mathrm{~nm}$ ) in a computer-controlled microplate reader (Bio-Rad, Tokyo, Japan) [29].

2.6. ELISA. IFN- $\gamma$, tumor necrosis factor- (TNF-) $\alpha$, interleukin- (IL-) 6, IL-4, and IL-10 levels in BALF were measured using a specific ELISA kit (Ready-Set-Go, eBioscience Inc., San Diego, CA) according to the manufacturer's instructions [25]. The product was tested and found to conform to all
eBioscience Inc. Quality Control release specifications. The lower limits of detection sensitivity of the kits for IFN- $\gamma$, TNF- $\alpha$, IL-6, IL-4, and IL-10 are 15, 8, 4, 4, and $30 \mathrm{pg} / \mathrm{mL}$, respectively. The intra- and interassay coefficients of variation for these ELISA were less than $10 \%$.

2.7. Flow Cytometric Analysis. Subpopulations of BAL cells were labeled with phycoerythrin- (PE-) labeled rat anti-CD8a (53.6) or rat-anti-CD1lb (M1/70) antibodies (BD Bioscience Pharmingen, San Diego, CA) and analyzed by a FACS Calibur 3S flow cytometer (Becton Dickinson, Sunnyvale, CA). Briefly, BAL cells were collected from 4 to 5 mice in each group on days 1 and 5 after infection and pooled by group. The BAL cells $\left(5 \times 10^{5}\right.$ cells) were suspended in $50 \mu \mathrm{L}$ of PBS, mixed with $20 \mu \mathrm{L}$ of each PE-labeled antibody, and incubated at $4^{\circ} \mathrm{C}$ for $30 \mathrm{~min}$. The cells were washed twice with PBS and at least 10,000 cells suspended in PBS were analyzed by FACS [25]. Finally, the data were analyzed with CellQuest software.

2.8. Determination of Anti-RSV Antibody Titer in Serum. The anti-RSV antibody titer in sera was determined by the ELISA test. RSV antigen was prepared from RSV-infected HEp-2 cells, and the control antigen was simultaneously prepared from uninfected cells. The ELISA test was performed according to the methods described elsewhere [30]. Briefly, 96well microtiter plates were coated with $100 \mu \mathrm{L}$ of RSV and control antigens diluted in PBS for $18 \mathrm{~h}$ at $4^{\circ} \mathrm{C}$. The plates were washed and blocked, and then test sera diluted with PBS were incubated at $37^{\circ} \mathrm{C}$ for $1 \mathrm{~h}$. Wells were washed extensively and incubated with peroxidase-conjugated rabbit anti-mouse IgG (DAKO A/S, Denmark) for $1 \mathrm{~h}$ at $37^{\circ} \mathrm{C}$. After washes with PBS, the enzyme reaction was performed and then the absorbance of the reaction mixture was measured. Anti-RSV antibody titer was determined by comparing the absorbance of control and RSV antigens.

2.9. Statistical Analysis. Statistical significance of differences between virus titers, cytokine levels, and anti-RSV-antibody levels evaluated using Student's $t$-test. A $P$ value of 0.05 or less was considered to be significant statistically.

\section{Results}

3.1. Effect of AF-08 on RSV Infection in Mice Exposed to TBBPA. We previously showed that exposure to $1 \%$ TBBPA for 28 days significantly increased pulmonary virus titers in RSV-infected mice and exacerbated pneumonia histologically [25]. Using this murine RSV infection model, we examined the effect of AF-08 on the exacerbation of RSV infection due to TBBPA exposure. Five-week-old mice were fed a control or 1\% TBBPA (w/w) diet for 28 days then intranasally infected with RSV. TBBPA-exposed mice showed no significant body weight loss or decrease in food consumption during the 28-day feeding period, when compared to control (data not shown). Then the mice with or without TBBPA exposure were intranasally infected with RSV. No significant weight loss was detected during the RSV infection phase (data not shown). On day 5 after infection, pulmonary 


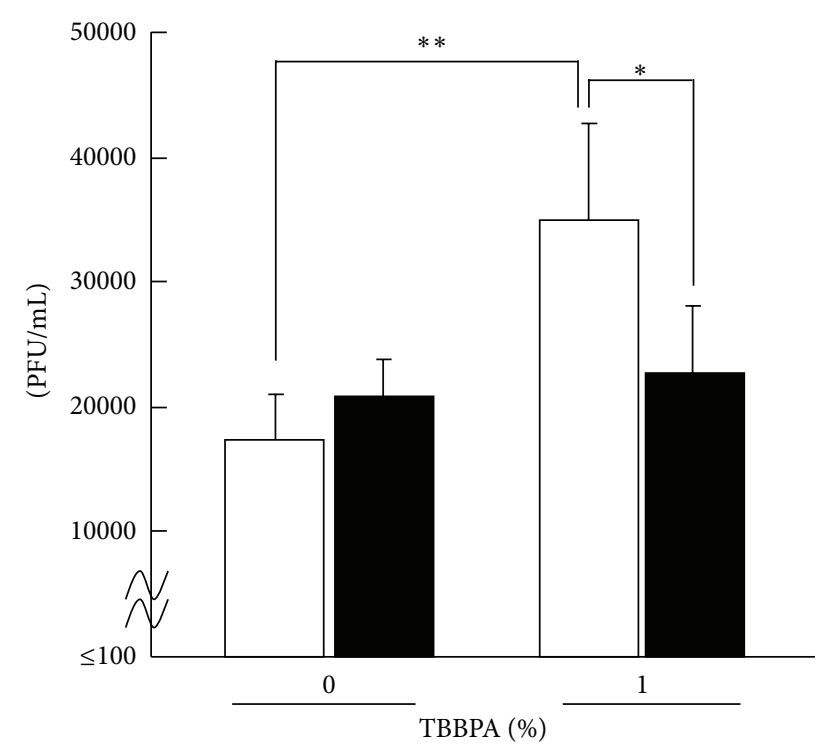

Control

AF-08

Figure 1: Effect of AF-08 on pulmonary viral titers in RSVinfected mice with TBBPA exposure. Seven mice in each group were fed a powdered diet mixed with 1\% TBBPA alone, 0.02\% AF-08 alone, or $1 \%$ TBBPA plus $0.02 \%$ AF- 08 or without $1 \%$ TBBPA and $0.02 \%$ AF-08 (control) for 4 weeks and then infected with RSV at $10^{6} \mathrm{PFU} / \mathrm{mouse}$. The lungs were removed on day 5 after infection, and virus titers in homogenized lungs were determined by a plaque assay. ${ }^{*} P<0.05$ and ${ }^{* *} P<0.001$ versus control by Student's $t$-test. Bars indicate standard errors.

viral titers in mice with TBBPA exposure were significantly higher than those in the control mice $(P<0.001$ versus control by Student's $t$-test, Figure 1). We confirmed the absence of toxicity and increase in pulmonary virus titers in RSVinfected mice exposed to TBBPA as described previously [25].

To examine the effect of AF-08 on the increase in pulmonary RSV titers by TBBPA exposure, we used $0.02 \%$ AF-08 mixed with a powdered diet with or without $1 \%$ TBBPA. In our previous studies, oral administration of AF08 at $10 \mathrm{mg} / \mathrm{kg}, 3$ times daily, was effective in reducing pulmonary influenza virus titers, resulting in the prolongation of survival times of influenza virus-infected mice [5] and in alleviating herpes simplex virus type-1 infection by enhancing delayed hypersensitivity to inactivate herpes simplex virus antigen [6]. These results indicate that $30 \mathrm{mg} / \mathrm{kg} /$ day may be a biologically active dose of AF-08 in mice. In addition, as the daily consumption of powered diet by a mouse is approximately $3 \mathrm{~g}$, a powdered diet containing $0.02 \% \mathrm{AF}-08$ was deduced from $0.6 \mathrm{mg} / \mathrm{mouse}$ of $20 \mathrm{~g} /$ day corresponding to the dose of AF-08 (30 mg/kg/day) and a daily dose of approximately $1500 \mathrm{mg} / \mathrm{kg} /$ day TBBPA was estimated. In the AF-08-treated and AF-08-treated plus TBBPA-exposed mice, there was no significant body weight loss or decrease in food consumption compared with the control (data not shown). As shown in Figure 1, 0.02\% AF-08 did not affect pulmonary virus titers in RSV-infected mice without TBBPA exposure but significantly reduced the virus titers in RSV-infected mice with TBBPA exposure $(P<0.05$ versus TBBPA alone by Student's $t$-test). The reduced titer was similar to those in RSV-infected mice without TBBPA exposure. Thus, AF-08 was effective in reducing RSV titers increased by TBBPA exposure to the normal levels without TBBPA exposure.

3.2. Anti-RSV Activity of AF-08 or TBBPA In Vitro. AF-08 and TBBPA were examined for their direct anti-RSV activity by plaque reduction assays using HEp- 2 cells (Table 1 ). In the assays, effective concentrations of ribavirin as a positive control were similar to the results reported by Konno et al. [31]. TBBPA did not significantly inhibit the plaque formation of RSV at $0.5 \mu \mathrm{g} / \mathrm{mL}(1 \mu \mathrm{M})$ to $16.3 \mu \mathrm{g} / \mathrm{mL}(30 \mu \mathrm{M})$, and no plaques were detected at $54.4 \mu \mathrm{g} / \mathrm{mL}(100 \mu \mathrm{M})$ because of the absence of more than $50 \%$ of uninfected cells. In the MTT assay, the percentage of viable cells in the presence of TBBPA at $54.4 \mu \mathrm{g} / \mathrm{mL}$ was $54.9 \pm 7.5 \%$. In the case of AF- 08 , a slight plaque reduction was observed at $1 \mu \mathrm{g} / \mathrm{mL}$. However, plaques were not detected at $5 \mu \mathrm{g} / \mathrm{mL}$, because of the absence of more than $50 \%$ of uninfected cells. The MTT assay of AF08 showed that the viable cells at AF- 08 of $5 \mu \mathrm{g} / \mathrm{mL}$ were $66.9 \pm 7.2 \%$. Thus the plaque reduction might be due to cytotoxicities of TBBPA and AF-08 rather than anti-RSV activity. TBBPA and AF-08 evidently did not show anti-RSV activity at their noncytotoxic concentrations.

3.3. Effects of AF-08 on Cytokine Production in BALF of RSVInfected Mice Exposed to TBBPA. It is known that the level of IFN- $\gamma$, a Thl cytokine, in BALF of RSV-infected mice is a sensitive marker of the severity of RSV infection of lungs $[11-13,25]$. Thus, we compared the IFN- $\gamma$ levels in the BALF from RSV-infected mice exposed to TBBPA with and without AF-08 treatment on days 1 and 5 after infection. As shown in Table 2, on day 5 after infection, TBBPA exposure significantly increased IFN- $\gamma$ level in the BALF of RSVinfected mice as compared with the control $(P<0.05$ versus control by Student's $t$-test), but its combination with AF-08 was significantly effective in blocking the increase prophylactically ( $P<0.05$ versus TBBPA by Student's $t$-test). The blocked IFN- $\gamma$ level was similar to the level in the BALF of RSV-infected mice treated with AF-08 alone. The IFN- $\gamma$ levels in the AF-08-treated mice in spite of TBBPA exposure were significantly lower than that in the control $(P<0.05$ versus control by Student's $t$-test). On day 1 after infection, IFN$\gamma$ levels with AF-08 alone and in combination with TBBPA tended to block the increase in the level with TBBPA alone, although the difference was not statistically significant. AF08 was effective in prophylactically blocking the increase in IFN- $\gamma$ level by TBBPA exposure in the lungs of RSV-infected mice.

To evaluate the effects of AF-08 on the immune system of RSV-infected mice with TBBPA exposure, the levels of proinflammatory cytokines (TNF- $\alpha$ and IL-6) and Th2 cytokines (IL-4 and IL-10) in BALF were also measured on days 1 and 5 after infection (Table 2). On day 1 after infection, TBBPA exposure significantly increased the TNF- $\alpha$ level in the BALF 
TABLE 1: Anti-RSV activity and cytotoxicity of TBBPA and AF-08 in vitro.

\begin{tabular}{|c|c|c|c|}
\hline & $\mu \mathrm{g} / \mathrm{mL}$ & $\begin{array}{l}\% \text { of viable cells } \\
\text { in MTT assay }\end{array}$ & $\begin{array}{l}\% \text { of plaque } \\
\text { numbers in } \\
\text { plaque } \\
\text { reduction assay }\end{array}$ \\
\hline \multirow{6}{*}{ ТВBPA } & 0 & 100 & 100 \\
\hline & 0.5 & $102.0 \pm 7.9$ & $95.2 \pm 24.4$ \\
\hline & 1.6 & $94.8 \pm 3.5$ & $87.1 \pm 8.4$ \\
\hline & 5.4 & $98.8 \pm 5.6$ & $91.9 \pm 9.7$ \\
\hline & 16.3 & $86.9 \pm 1.3$ & $80.6 \pm 10.1$ \\
\hline & 54.4 & $54.9 \pm 7.5$ & $++^{*}$ \\
\hline \multirow{7}{*}{ AF-08 } & 0 & 100 & 100 \\
\hline & 0.1 & - & $93.8 \pm 8.1$ \\
\hline & 0.5 & - & $82.8 \pm 31.2$ \\
\hline & 1 & - & $74.2 \pm 3.6$ \\
\hline & 5 & $66.9 \pm 7.2$ & $++^{*}$ \\
\hline & 20 & $56.5 \pm 5.3$ & - \\
\hline & 40 & $33.0 \pm 1.7$ & - \\
\hline \multirow{5}{*}{ Ribavirin } & 0 & - & 100 \\
\hline & 1 & - & $75.0 \pm 12.5$ \\
\hline & 5 & - & $29.2 \pm 20.1$ \\
\hline & 10 & - & $10.4 \pm 7.5$ \\
\hline & 15 & - & 0 \\
\hline
\end{tabular}

Values are mean \pm standard deviation (SD) of the mean of three to five independent experiments.

$(-)$ indicates that experiments were not done.

${ }^{*} \%$ of plaque numbers could not be determined, because of the absence of more than $50 \%$ of uninfected and stained cells from the surface of dishes.

of RSV-infected mice $(P<0.05$ versus control by Student's $t$ test). The combination with AF-08 significantly blocked the increase $(P<0.01$ versus TBBPA by Student's $t$-test), and the prophylactically blocked level was similar to that in RSVinfected mice treated with AF-08 alone, although the former level in the combination was significantly lower than that in the control $(P<0.01$ versus control by Student's $t$-test $)$, while the latter level in AF-08 alone was not. In the case of IL-6, AF-08 also seemed to be effective in reducing the IL-6 levels in RSV-infected mice despite the TBBPA exposure on day 1 . On day 5 , the levels of TNF- $\alpha$ were much lower than those on day 1 and the effects of AF-08 on TNF- $\alpha$ level were not clear. However, AF-08 significantly reduced IL-6 levels in RSV-infected mice despite TBBPA exposure $(P<0.01$ versus control or TBBPA by Student's $t$-test). AF-08 was effective in blocking TBBPA-dependent exacerbation in proinflammatory cytokine response. All RSV infections investigated in these studies caused a rise in levels of proinflammatory cytokines. In the cases of Th2 cytokines, IL-4 levels were low on days 1 and 5 after infection and the effects of AF-08 and/or TBBPA were not obvious. The IL-10 level in the RSVinfected control mice was not significantly affected by TBBPA exposure and/or AF-08 treatment, excepting the significant reduction of IL-10 level in AF-08-treated mice $(P<0.05$ versus control by Student's $t$-test). Thus, AF-08 was mainly

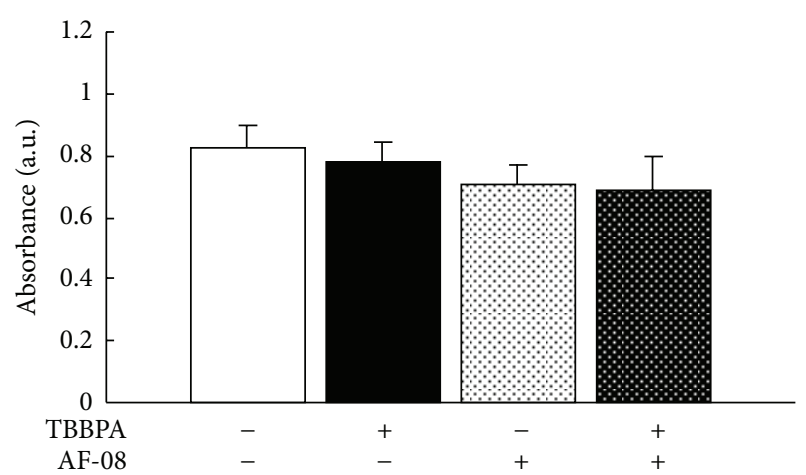

FIGURE 2: Effects of AF-08 on anti-RSV antibody production in RSV-infected mice with TBBPA exposure. Five mice in each group were fed a powdered diet mixed with $1 \%$ TBBPA alone, $0.02 \%$ AF08 alone, or $1 \%$ TBBPA plus $0.02 \%$ AF- 08 or without $1 \%$ TBBPA and $0.02 \%$ AF-08 (control) for 4 weeks and then infected with RSV at $10^{6} \mathrm{PFU} / \mathrm{mouse}$. On day 5 after infection, the amounts of anti-RSV antibody in sera of RSV-infected mice were determined by ELISA. Bars indicate standard errors.

effective in controlling the rise of IFN- $\gamma$, TNF- $\alpha$, and IL-6 levels caused by TBBPA exposure in RSV-infected mice.

3.4. Effect of AF-08 on Production of Anti-RSV Antibody in Mice Exposed to TBBPA. The effect of AF-08 on production of antibodies to RSV was compared in sera prepared from RSV-infected mice with and without TBBPA exposure. As shown in Figure 2, TBBPA exposure alone, AF-08 treatment alone, and their combination did not affect the anti-RSV antibody levels in sera of RSV-infected mice on day 5 after infection. Neither AF-08 treatment nor TBBPA exposure affected the production of anti-RSV antibody in RSV-infected mice.

3.5. Effect of AF-08 on Subpopulations of Immune Cells in $B A L$ from RSV-Infected Mice. To evaluate the effect of AF08 on pulmonary immune responses in RSV-infected mice with TBBPA exposure, the subpopulations of immune cells in BALF were analyzed by flow cytometry on days 1 and 5 after infection (Table 3 ). Total numbers of BAL cells collected from an RSV-infected mouse in each group were 2.2 to 2.8 $\times 10^{5}$ cells, and there were no significant differences in the numbers of BAL cells in control, AF-08-treated, TBBPAtreated, and AF-08 plus TBBPA-treated groups (data not shown). On day $1, \mathrm{CD} 1 \mathrm{~b}^{+}$cells including macrophages and dendritic cells were dominant in BAL cells in each group $(87.8 \%, 81.2 \%, 87.1 \%$, and $87.3 \%)$, but the percentages of $\mathrm{CD} 1 \mathrm{~b}^{+}$cells evidently decreased on day $5(28.4 \%, 13.5 \%$, $22.2 \%$, and $17.6 \%)$. On the other hand, the percentages of $\mathrm{CD}^{+} \mathrm{a}^{+}$cells $(4.0 \%, 4.1 \%, 5.5 \%$, and $4.5 \%)$ in each group on day 1 markedly increased on day $5(50.8 \%, 62.5 \%, 69.1 \%$, and 49.1\%). However, TBBPA exposure and/or AF-08 treatment did not markedly affect the percentages of CD11b ${ }^{+}$and CD8a ${ }^{+}$ cells in the controls on days 1 and 5 . The ratios of percentages of $\mathrm{CD}_{11 b^{+}}$cells in the groups of AF-08, TBBPA, and AF-08 and TBBPA against those in the control were 0.92 to 0.99 on 
TABLE 2: Effect of AF-08 on cytokine concentrations in BALF from RSV-infected mice exposed or unexposed to TBBPA.

\begin{tabular}{|c|c|c|c|c|}
\hline \multirow{2}{*}{ Cytokines } & \multicolumn{4}{|c|}{ Concentrations $\pm \mathrm{SE}(\mathrm{pg} / \mathrm{mL})$ in BALF } \\
\hline & Control & AF-08 & TВBРA & $\mathrm{TBBPA}+\mathrm{AF}-08$ \\
\hline \multicolumn{5}{|l|}{ Day 1} \\
\hline IFN- $\gamma$ & $23.9 \pm 4.3$ & $30.0 \pm 5.3$ & $56.2 \pm 10.9$ & $33.1 \pm 6.8$ \\
\hline TNF- $\alpha$ & $271.6 \pm 25.2$ & $148.1 \pm 76.2^{\mathrm{c}}$ & $339.0 \pm 22.3^{\mathrm{a}}$ & $122.1 \pm 17.9^{\mathrm{b}, \mathrm{d}}$ \\
\hline IL-6 & $1076.1 \pm 150.9$ & $848.7 \pm 341.7$ & $926.1 \pm 183.8$ & $<470.0 \pm 305.3$ \\
\hline IL-4 & $<28.0 \pm 11.4$ & $<20.0 \pm 0.0$ & $<28.0 \pm 11.4$ & $<20.0 \pm 0.0$ \\
\hline IL-10 & $144.0 \pm 63.1$ & $149.4 \pm 45.4$ & $208.7 \pm 69.9$ & $102.3 \pm 35.6$ \\
\hline \multicolumn{5}{|l|}{ Day 5} \\
\hline IFN- $\gamma$ & $2557.4 \pm 117.4$ & $1507.6 \pm 491.9^{\mathrm{a}, \mathrm{c}}$ & $3348.3 \pm 410.8^{\mathrm{a}}$ & $1108.3 \pm 511.3^{\mathrm{a}, \mathrm{c}}$ \\
\hline TNF- $\alpha$ & $<40.0$ & $<40.0$ & $<40.0$ & $<40.0$ \\
\hline IL-6 & $111.8 \pm 26.7$ & $<28.7 \pm 15.0^{\mathrm{b}}$ & $<67.7 \pm 60.9$ & $<20.0 \pm 0.0^{\mathrm{b}}$ \\
\hline IL-4 & $<20.0 \pm 0.0$ & $<20.0 \pm 0.0$ & $<20.6 \pm 1.0$ & $<36.4 \pm 28.3$ \\
\hline IL-10 & $197.4 \pm 37.4$ & $118.2 \pm 9.5^{\mathrm{a}}$ & $195.5 \pm 99.2$ & $110.7 \pm 68.1$ \\
\hline
\end{tabular}

BALF was collected from 3-4 mice in each group on day 1 and day 5 after infection. Each BALF was analyzed to determine cytokine concentrations by ELISA. ${ }^{\mathrm{a}} P<0.05$ versus control by Student's $t$-test. ${ }^{\mathrm{b}} P<0.01$ versus control by Student's $t$-test. ${ }^{\mathrm{c}} P<0.05$ versus TBBPA by Student's $t$-test. ${ }^{\mathrm{d}} P<0.01$ versus TBBPA by Student's $t$-test.

TABLE 3: Effect of AF-08 on subpopulations in BAL cells of RSVinfected mice exposed or unexposed to TBBPA.

\begin{tabular}{lcccc}
\hline \multirow{2}{*}{ Subpopulation } & \multicolumn{4}{c}{ \% of total cell populations } \\
& Control & AF-08 & TBBPA & TBBPA + AF-08 \\
\hline Day 1 & & & & \\
CD11b & 87.8 & 81.2 & 87.1 & 87.3 \\
${\text { CD } 8 a^{+}}^{+}$ & 4.0 & 4.1 & 5.5 & 4.5 \\
Day 5 & & & & \\
${\text { CD } 11 b^{+}}_{\text {CD }^{+} \mathrm{a}^{+}}^{28.4}$ & 23.5 & 22.2 & 17.6 \\
\hline
\end{tabular}

BAL cells were collected from 3-4 mice in each group on day 1 and day 5 after infection and pooled by group. The subpopulations of pooled BAL cells were analyzed by FACS.

day 1 and 0.62 to 0.83 on day 5 . The ratios of percentages of CD8a ${ }^{+}$cells in the groups of AF-08, TBBPA, and AF08 and TBBPA against those in the control were 1.03 to 1.38 on day 1 and 0.97 to 1.36 on day 5 . AF-08 seemed to be effective in reducing the ratio of pulmonary $\mathrm{CD} 8 \mathrm{a}^{+}$cells in $\mathrm{RSV}$-infected mice with TBBPA exposure.

\section{Discussion}

Propolis is a natural product known worldwide as a folk medicine and used as a dietary supplement because of its wide range of reported biological activities [1-6]. The chemical composition of propolis depends on the area and vegetation where it was harvested [6]. It has been administered orally for improvement of the symptoms of viral infection $[5,6]$. However, there has been little research to elucidate the theoretical bases for the clinical efficacy of propolis. In this study, we characterized the basis of the biological efficacy of propolis on the exacerbation of pneumonia caused by TBBPA exposure in RSV-infected mice and found that a Brazilian propolis, AF-08, is effective in alleviating pneumonia exacerbated by
TBBPA exposure in RSV-infected mice through limitation of excess cellular immune responses. Previously we showed that the oral administration of AF-08 is effective in ameliorating skin herpes symptoms in mice through activation of a delayed type hypersensitive reaction based on Th1 immunity as a major immune defense system in intradermal HSV-1 infection [6, 32]. In this study, AF-08 did not exhibit anti-RSV activity in a plaque reduction assay (Table 1 ) or affect virus titer in the lungs of RSV-infected mice (Figure 1). Thus, AF08 may be characterized as possessing immunomodulatory activity based on cellular immunity. It may be worthwhile to further investigate the immunologically active compounds that are involved in AF-08.

TBBPA exposure significantly increased virus titers in the lungs of RSV-infected mice, while coadministration of AF-08 with TBBPA blocked this increase; virus titer in these mice was not different from that observed for controls (Figure 1). However, neither TBBPA nor AF-08 exhibited direct anti-RSV activity in vitro (Table 1). Thus, the activated immunological responses to eliminate infectious RSV particles and RSV-infected cells in the lungs of mice are probably responsible for the alleviation of pneumonia exacerbated by TBBPA exposure. Perinatal exposure to BFRs was also shown to exacerbate pneumonia in RSV-infected offspring mice with an increase in pulmonary viral titers and there was a correlation between the severity of RSV infection in the offspring mice and the dose exposed to pregnant mice $[12,13]$. The dose $(30 \mathrm{mg} / \mathrm{kg} /$ day $)$ of AF-08 given to mice was analogous to that ingested by humans [6]. From the viewpoint of environmental medicines, it is possible that oral administration of AF-08 not only to adults but also to children born from women exposed to BFRs such as TBBPA is prophylactically useful to prevent the exacerbation of RSV infection.

IFN $-\gamma$ has been reported to be a potent stimulator of lymphocyte migration into tissues and a major mediator of lymphocyte recruitment in Th1 immunity [33]. The level of 
IFN- $\gamma$, a marker of pneumonia, has been shown to reach a maximum on day 5 after infection in RSV-infected mice, and its production was enhanced in TBBPA-exposed mice [25]. In this study, the elevation of the IFN- $\gamma$ level by TBBPA exposure in the lungs of RSV-infected mice on day 5 was confirmed. The elevation by TBBPA on day 5 was significantly reduced by AF-08 in combination with TBBPA (Table 2). Also, levels of both proinflammatory cytokines (TNF- $\alpha$ and IL-6) in RSVinfected mice with TBBPA exposure on day 1 were reduced by AF-08 in the combination with TBBPA (Table 2). The reductions in TNF- $\alpha$ and IL- 6 by AF-08 were prominent early in the infection, suggesting that the beneficial effect of AF-08 was due to the suppression of inflammation early after infection. In addition, AF-08 was effective in markedly reducing the IFN- $\gamma$ level on day 5 (Table 2). Thus, the suppression of proinflammatory cytokine production by AF08 early in the infection was associated with weakening inflammation in the lungs of infected mice and reducing the infiltrated cell population quantitatively and/or qualitatively, resulting in suppression of the overreaction of Thl immune response correlated with the elevation of IFN- $\gamma$ level in the infected lungs. Actually, AF-08 alone was effective in reducing the levels of proinflammatory cytokines (TNF- $\alpha$ and IL-6) in RSV-infected mice on day 1 and the level of IFN$\gamma$ on day 5 . Therefore, the suppression of proinflammatory cytokine productions by AF-08 in RSV-infected mice is probably responsible for the reduction of the IFN- $\gamma$ level in the combination leading the alleviation of pneumonia. Also, as the excess productions of proinflammatory cytokines by TBBPA in RSV-infected mice caused severe inflammatory response, the suppression of the cytokines by AF-08 was suggested to be effective in blocking an imbalance in the inflammatory response caused by TBBPA in RSV-infected mice. TBBPA was reported to activate the MAP kinases and protein kinase $C[34,35]$. AF-08 may be effective in suppressing their activation in signal transduction systems involved in the overexpression of proinflammatory cytokines.

TBBPA exposure failed to significantly elevate the levels of Th2 cytokines (IL-4 and IL-10) in RSV-infected mice on either day 1 or day 5 , and there were no significant reductions of the Th2 cytokine levels by AF-08 in combination with TBBPA (Table 2). TBBPA and AF-08 did not noticeably affect Th2 cytokine (IL-4 and IL-10) levels in RSV-infected mice. It was reported that an imbalance of Thl/2 cytokines in the immune system is involved in the severity of RSV-induced disease [36]. The strong production of Thl cytokines, such as IFN- $\gamma$, exacerbating RSV infection might disorder Th2 cytokine production in infected mice after TBBPA exposure and/or AF-08 treatment. In RSV-infected mice, TBBPA and/or AF-08 had no effect on the production of anti-RSV antibody (Figure 2), suggesting that neither TBBPA nor AF08 mainly affects humoral immunity. However, TBBPA and AF-08 affected cellular immunity induced by Th1 immune response associated with IFN- $\gamma$ production in RSV-infected mice, as described before. It is possible that TBBPA produced an imbalance between the Th1 and Th2 immunity caused by excessive Th1-dominant immune response in RSV-infected mice and AF-08 blocked the imbalance by limiting the excessive Thl immunity.
FACS analysis showed that $\mathrm{CD} 1 \mathrm{~b}^{+}$cells involving macrophages and dendritic cells and $\mathrm{CD}^{+}$cells involving cytotoxic $\mathrm{T}$ cells were dominant in BAL cells from RSV-infected mice in spite of TBBPA exposure and/or AF-08 treatment on days 1 and 5, respectively (Table 3 ). $\mathrm{CD}_{11 b^{+}}$cells and CD ${ }^{+}$ cells are the major producers of proinflammatory cytokines and IFN- $\gamma$, respectively. Thus, the results of FACS analysis were consistent in the elevations of proinflammatory cytokine production on day 1 and IFN- $\gamma$ production on day 5 (Table 2 ). TBBPA exposure and/or AF-08 treatment had no remarkable effect on the percentages of $\mathrm{CD} 1 \mathrm{~b}^{+}$cells and $\mathrm{CD}^{+}$cells in BAL cells from RSV-infected mice (Table 3). However, while TBBPA was effective in elevating the productions of proinflammatory cytokines and IFN- $\gamma$, AF-08 was effective in reducing their productions (Table 2). TBBPA has been suggested to induce a qualitative change in immune cells that work in the early phase of RSV infection [25]. Thus, AF-08 also might induce a qualitative change in immune cells in the lungs of RSV-infected mice.

We characterized the experimental bases of Brazilian propolis AF-08 treatment using an RSV infection model in mice. Together, all results indicate that propolis AF08 showed immunological activity against intranasal RSV infection in mice. In particular, the immunological activity associated with the limitation of IFN- $\gamma$ production inducing Th1 immunity in mice may contribute to the elucidation of various pharmacological actions of propolis AF-08 in health and disease.

\section{Conflict of Interests}

No potential conflict of interest was disclosed.

\section{Acknowledgments}

The authors thank Ms. Yukiko Shimoda and Ms. Yuko Yanagida for their excellent technical assistance and Ms. Katherine Ono for her editorial assistance. This study was partly supported by Grants-in-Aid for Scientific Research (no. 23590161) and for Young Scientists (no. 24790182) from Japan Society for the Promotion of Science and Health and Labour Sciences Research Grants [Research on Risk of Chemical Substances] from Ministry of Health, Labour and Welfare of Japan.

\section{References}

[1] V. Bankova, R. Christov, A. Kujumgiev, M. C. Marcucci, and S. Popov, "Chemical composition and antibacterial activity of Brazilian propolis," Zeitschrift fur Naturforschung C, vol. 50, no. 3-4, pp. 167-172, 1995.

[2] A. H. Banskota, Y. Tezuka, and S. Kadota, "Recent progress in pharmacological research of propolis," Phytotherapy Research, vol. 15, no. 7, pp. 561-571, 2001.

[3] G. A. Burdock, "Review of the biological properties and toxicity of bee propolis (propolis)," Food and Chemical Toxicology, vol. 36, no. 4, pp. 347-363, 1998.

[4] M. L. Khalil, "Biological activity of bee propolis in health and disease," Asian Pacific Journal of Cancer Prevention, vol. 7, no. 1, pp. 22-31, 2006. 
[5] T. Shimizu, A. Hino, A. Tsutsumi, K. P. Yong, W. Watanabe, and M. Kurokawa, "Anti-influenza virus activity of propolis in vitro and its efficacy against influenza infection in mice," Antiviral Chemistry and Chemotherapy, vol. 19, no. 1, pp. 7-13, 2008.

[6] M. Kurokawa, T. Shimizu, Y. Takeshita et al., "Efficacy of Brazilian propolis against herpes simplex virus type 1 infection in mice and their modes of antiherpetic efficacies," Evidence-based Complementary and Alternative Medicine, vol. 2011, Article ID 976196, 9 pages, 2011.

[7] N. E. MacDonald, C. B. Hall, and S. C. Suffin, "Respiratory syncytial viral infection in infants with congenital heart disease," The New England Journal of Medicine, vol. 307, no. 7, pp. 397400, 1982.

[8] P. L. Collins, R. M. Chanock, and B. R. Murphy, "Respiratory syncytial virus," in Fields Virology, D. M. Knipe and P. M. Howley, Eds., pp. 1443-1485, Lippincott Williams \& Wilkins, Philadelphia, Pa, USA, 2001.

[9] C. J. Holberg, A. L. Wright, F. D. Martinez, C. G. Ray, L. M. Taussig, and M. D. Lebowitz, "Risk factors for respiratory syncytial virus-associated lower respiratory illnesses in the first year of life," American Journal of Epidemiology, vol. 133, no. 11, pp. 1135-1151, 1991.

[10] F. Morales, M. A. Calder, and J. M. Inglis, "A study of respiratory infections in the elderly to assess the role of respiratory syncytial virus," Journal of Infection, vol. 7, no. 3, pp. 236-247, 1983.

[11] W. Watanabe, T. Shimizu, A. Hino, and M. Kurokawa, "A new assay system for evaluation of developmental immunotoxicity of chemical compounds using respiratory syncytial virus infection to offspring mice," Environmental Toxicology and Pharmacology, vol. 25, no. 1, pp. 69-74, 2008.

[12] W. Watanabe, T. Shimizu, A. Hino, and M. Kurokawa, "Effects of decabrominated diphenyl ether (DBDE) on developmental immunotoxicity in offspring mice," Environmental Toxicology and Pharmacology, vol. 26, no. 3, pp. 315-319, 2008.

[13] W. Watanabe, T. Shimizu, R. Sawamura, A. Hino, K. Konno, and M. Kurokawa, "Functional disorder of primary immunity responding to respiratory syncytial virus infection in offspring mice exposed to a flame retardant, decabrominated diphenyl ether, perinatally," Journal of Medical Virology, vol. 82, no. 6, pp. 1075-1082, 2010.

[14] L. S. Birnbaum and D. F. Staskal, "Brominated flame retardants: cause for concern?" Environmental Health Perspectives, vol. 112, no. 1, pp. 9-17, 2004.

[15] M. Lundgren, P. O. Darnerud, J. Blomberg, G. Friman, and N.G. Ilbäck, "Polybrominated diphenyl ether exposure suppresses cytokines important in the defence to coxsackievirus B3 infection in mice," Toxicology Letters, vol. 184, no. 2, pp. 107-113, 2009.

[16] S. Pullen, R. Boecker, and G. Tiegs, “The flame retardants tetrabromobisphenol A and tetrabromobisphenol A-bisallylether suppress the induction of interleukin-2 receptor $\alpha$ chain (CD25) in murine splenocytes," Toxicology, vol. 184, no. 1, pp. 11-22, 2003.

[17] R. Teshima, R. Nakamura, R. Nakamura, A. Hachisuka, J.-I. Sawada, and M. Shibutani, "Effects of exposure to decabromodiphenyl ether on the development of the immune system in rats," Journal of Health Science, vol. 54, no. 4, pp. 382-389, 2008.

[18] R. Ceccatelli, O. Faass, M. Schlumpf, and W. Lichtensteiger, "Gene expression and estrogen sensitivity in rat uterus after developmental exposure to the polybrominated diphenylether PBDE 99 and PCB," Toxicology, vol. 220, no. 2-3, pp. 104-116, 2006.
[19] S. N. Kuriyama, A. Wanner, A. A. Fidalgo-Neto, C. E. Talsness, W. Koerner, and I. Chahoud, "Developmental exposure to lowdose PBDE-99: tissue distribution and thyroid hormone levels," Toxicology, vol. 242, no. 1-3, pp. 80-90, 2007.

[20] T. A. McDonald, "Polybrominated diphenylether levels among United States residents: daily intake and risk of harm to the developing brain and reproductive organs," Integrated Environmental Assessment and Management, vol. 1, no. 4, pp. 343-354, 2005.

[21] H. Lilienthal, A. Hack, A. Roth-Härer, S. W. Grande, and C. E. Talsness, "Effects of developmental exposure to 2,2,4,4, 5pentabromodiphenyl ether (PBDE-99) on sex steroids, sexual development, and sexually dimorphic behavior in rats," Environmental Health Perspectives, vol. 114, no. 2, pp. 194-201, 2006.

[22] P. A. Martin, G. J. Mayne, S. J. Bursian et al., "Immunotoxicity of the commercial polybrominated diphenyl ether mixture DE-71 in ranch mink (Mustela vison)," Environmental Toxicology and Chemistry, vol. 26, no. 5, pp. 988-997, 2007.

[23] T. Reistad and E. Mariussen, "A commercial mixture of the brominated flame retardant pentabrominated diphenyl ether (DE-71) induces respiratory burst in human neutrophil granulocytes in vitro," Toxicological Sciences, vol. 87, no. 1, pp. 57-65, 2005.

[24] D. Fischer, K. Hooper, M. Athanasiadou, I. Athanassiadis, and A. Bergman, "Children show highest levels of polybrominated diphenyl ethers in a California family of four: a case study," Environmental Health Perspectives, vol. 114, no. 10, pp. 1581-1584, 2006.

[25] W. Watanabe, T. Shimizu, R. Sawamura et al., "Effects of tetrabromobisphenol A, a brominated flame retardant, on the immune response to respiratory syncytial virus infection in mice," International Immunopharmacology, vol. 10, no. 4, pp. 393-397, 2010.

[26] N. Masutomi, M. Shibutani, H. Takagi, C. Uneyama, and M. Hirose, "Dietary influence on the impact of ethinylestradiolinduced alterations in the endocrine/reproductive system with perinatal maternal exposure," Reproductive Toxicology, vol. 18, no. 1, pp. 23-33, 2004.

[27] M. Kurokawa, M. Tsurita, J. Brown, Y. Fukuda, and K. Shiraki, "Effect of interleukin-12 level augmented by Kakkon-to, a herbal medicine, on the early stage of influenza infection in mice," Antiviral Research, vol. 56, no. 2, pp. 183-188, 2002.

[28] M. Kurokawa, H. Ochiai, K. Nagasaka et al., "Antiviral traditional medicines against herpes simplex virus (HSV-1), poliovirus, and measles virus in vitro and their therapeutic efficacies for HSV-1 infection in mice," Antiviral Research, vol. 22, no. 2-3, pp. 175-188, 1993.

[29] W. Watanabe, K. Konno, K. Ijichi, H. Inoue, T. Yokota, and S. Shigeta, "MTT colorimetric assay system for the screening of antiorthomyxo- and anti-paramyxoviral agents," Journal of Virological Methods, vol. 48, no. 2-3, pp. 257-265, 1994.

[30] K. Nagasaka, M. Kurokawa, M. Imakita, K. Terasawa, and K. Shiraki, "Efficacy of Kakkon-to, a traditional herb medicine, in herpes simplex virus type 1 infection in mice," Journal of Medical Virology, vol. 46, no. 1, pp. 28-34, 1995.

[31] K. Konno, R. Sawamura, Y. Sun et al., "Antiviral activities of diarylheptanoids isolated from Alpinia officinarum against respiratory syncytial virus, poliovirus, measles virus, and herpes simplex virus type 1 in vitro," Natural Product Communications, vol. 6, no. 12, pp. 1881-1884, 2011.

[32] A. A. Nash, H. J. Field, and R. Quartey-Papafio, "Cell-mediated immunity in herpes simplex virus-infected mice: induction, 
characterization and antiviral effects of delayed type hypersensitivity," Journal of General Virology, vol. 48, no. 2, pp. 351-357, 1980.

[33] T. B. Issekutz, J. M. Stoltz, and V. P. d. Meide, "Lymphocyte recruitment in delayed-type hypersensitivity. The role of IFN$\gamma$," Journal of Immunology, vol. 140, no. 9, pp. 2989-2993, 1988.

[34] L. Canesi, L. C. Lorusso, C. Ciacci, M. Betti, and G. Gallo, "Effects of the brominated flame retardant tetrabromobisphenol-A (TBBPA) on cell signaling and function of Mytilus hemocytes: involvement of MAP kinases and protein kinase C," Aquatic Toxicology, vol. 75, no. 3, pp. 277-287, 2005.

[35] T. Reistad and E. Mariussen, "A commercial mixture of the brominated flame retardant pentabrominated diphenyl ether (DE-71) induces respiratory burst in human neutrophil granulocytes in vitro," Toxicological Sciences, vol. 87, no. 1, pp. 57-65, 2005.

[36] M. E. Waris, C. Tsou, D. D. Erdman, S. R. Zaki, and L. J. Anderson, "Respiratory syncytial virus infection in BALB/c mice previously immunized with formalin-inactivated virus induces enhanced pulmonary inflammatory response with a predominant Th2-like cytokine pattern," Journal of Virology, vol. 70, no. 5, pp. 2852-2860, 1996. 


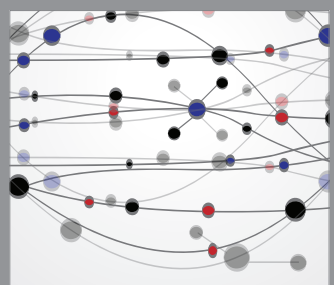

The Scientific World Journal
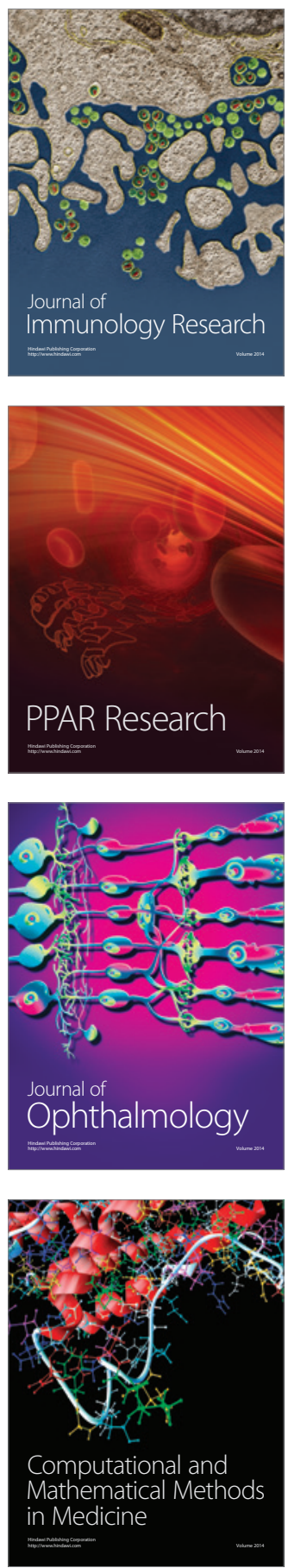

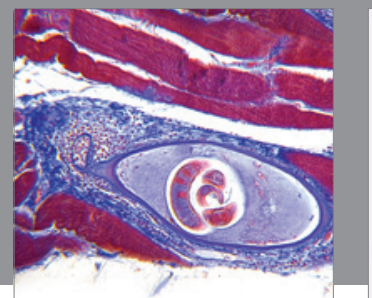

Gastroenterology

Research and Practice
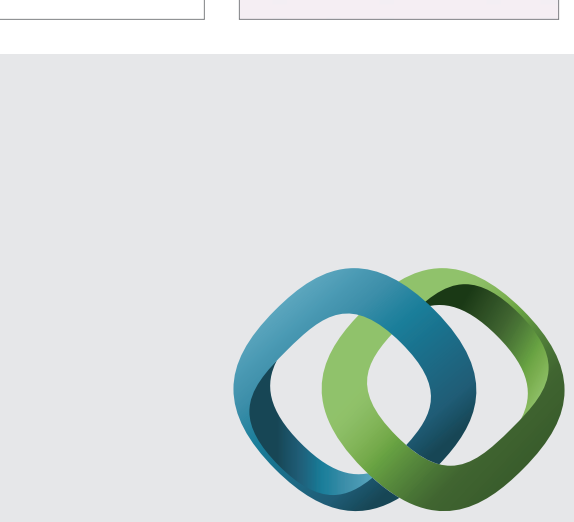

\section{Hindawi}

Submit your manuscripts at

http://www.hindawi.com
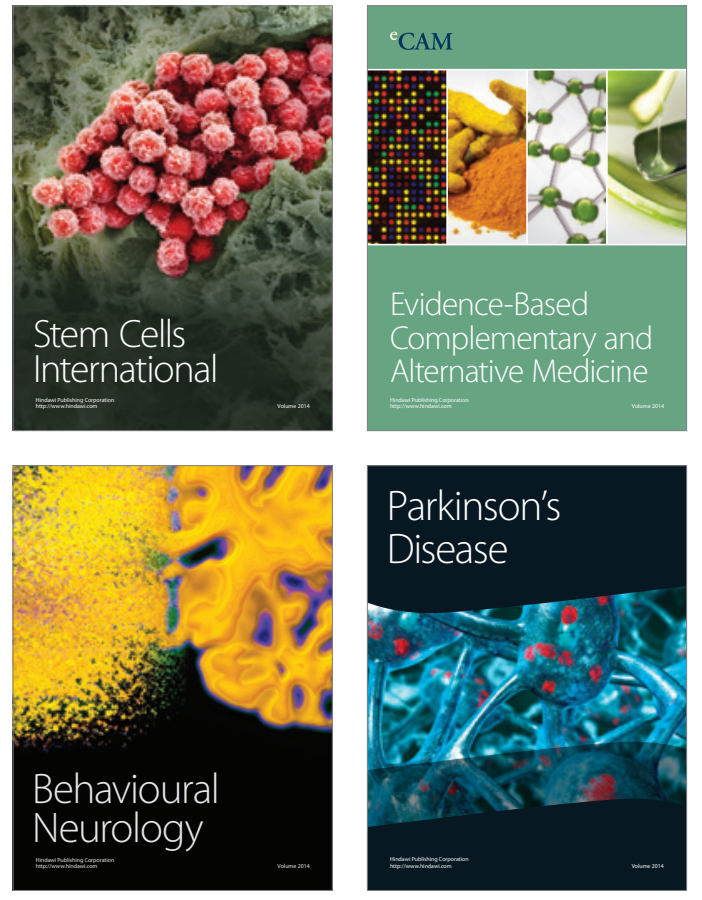
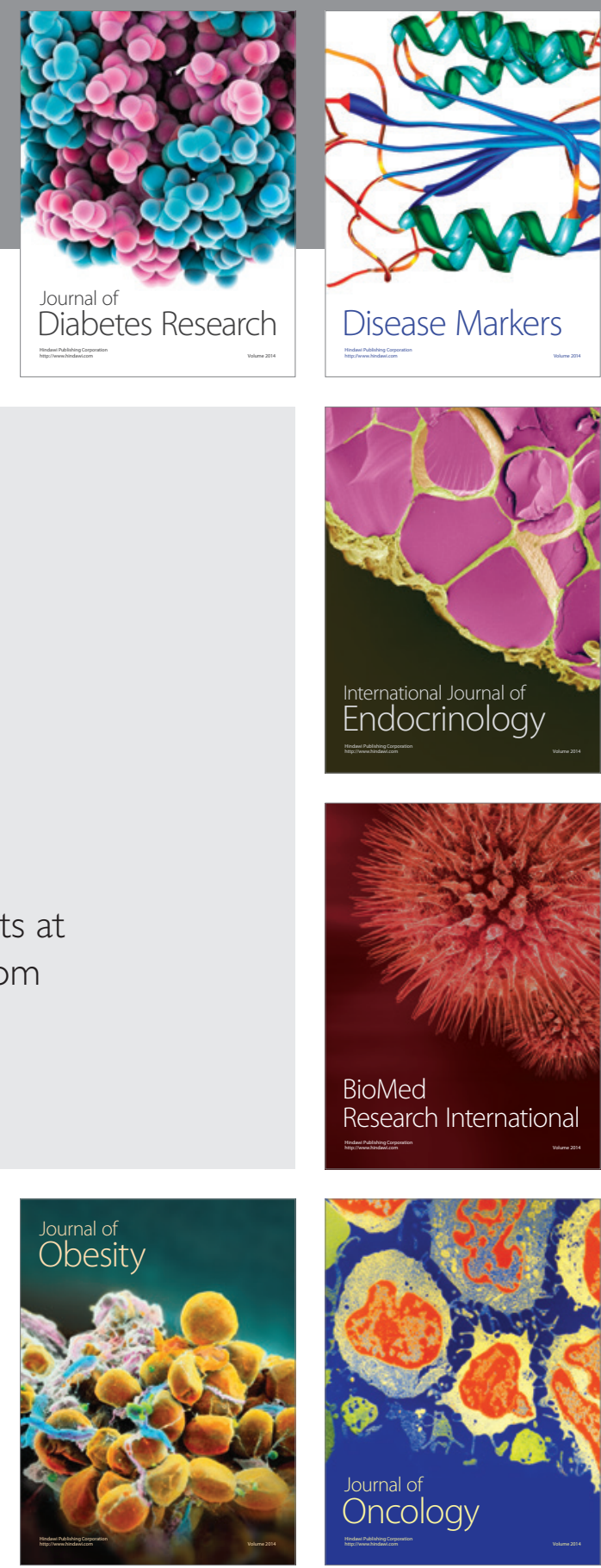

Disease Markers
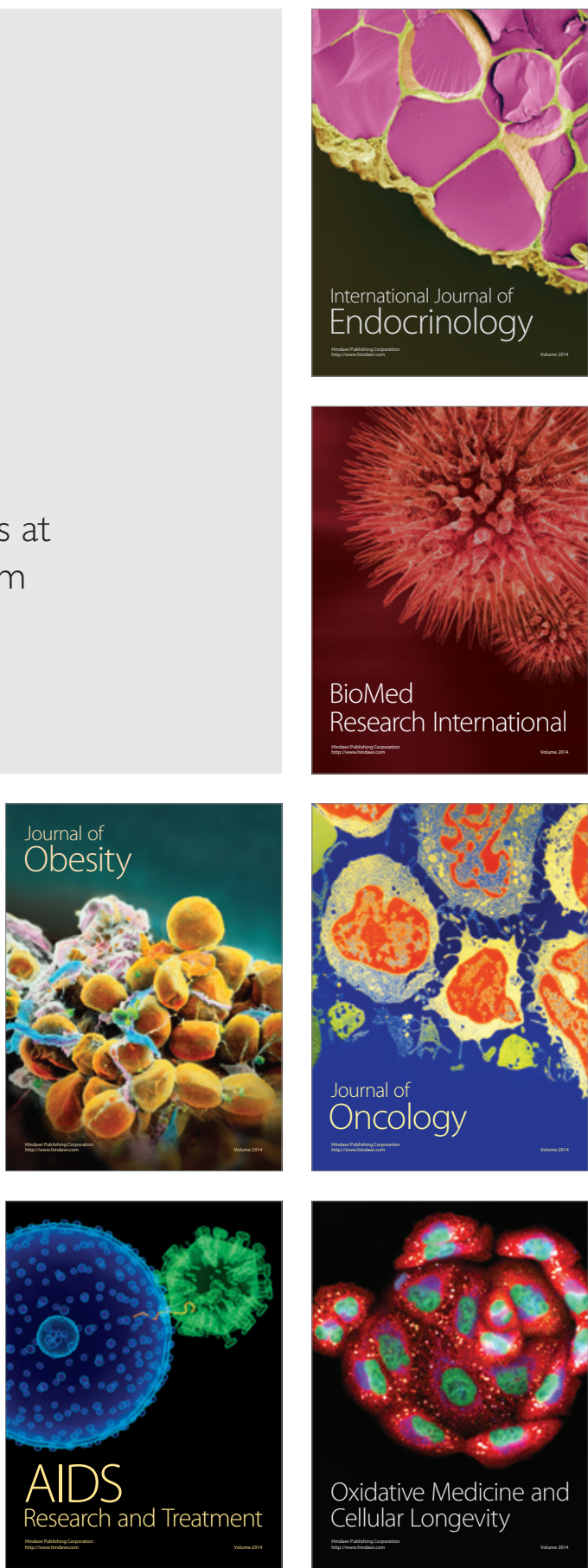